\title{
Expression and clinical significance of p73 in Wilms' tumor in children
}

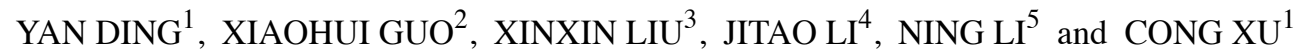 \\ ${ }^{1}$ Department of Pediatrics (II), People's Hospital of Rizhao, Rizhao, Shandong 276800; \\ ${ }^{2}$ Department of Child Health Care, The Third People's Hospital of Linyi, Linyi, Shandong 276000; \\ ${ }^{3}$ Department of Spine Surgery, The Affiliated Central Hospital of Qingdao University, Qingdao Cancer Hospital, \\ Qingdao, Shandong 266001; ${ }^{4}$ Electrocardiogram Room and ${ }^{5}$ Department of Pathology, \\ The People's Hospital of Zhangqiu Area, Jinan, Shandong 250200, P.R. China
}

Received November 8, 2018; Accepted March 21, 2019

DOI: 10.3892/ol.2019.10249

\begin{abstract}
Expression and clinical significance of p73 in children with Wilms' tumor (WT) were investigated. A retrospective analysis was carried out on 50 children diagnosed with WT in the People's Hospital of Rizhao, from July 2013 to January 2015 (study group), and 20 healthy children with similar age and sex who received health examinations in the same hospital during the same period (control group). The relative expression of p73 mRNA in the peripheral blood of each group was detected by reverse transcription-quantitative PCR (RT-qPCR) in order to study the association between p73 and the clinicopathological parameters of WT, as well as the impact of p73 on the patient prognosis. The two groups were not statistically different in respect to the clinical data of patients $(\mathrm{P}>0.05)$; the expression level of $\mathrm{p} 73$ in the blood samples of the study group was significantly higher than that of the control group $(\mathrm{t}=11.44, \mathrm{P}<0.01)$; the expression of p73 in the study group was associated with factors, including tumor size, pathological type and lymphatic metastasis. Considering the mean value of the expression of p73 (3.32) as the boundary, the patients with expression value of $\mathrm{p} 73<3.32$ were studied as the low-expression group, and patients with expression value of p73 $>3.32$ comprised the high-expression group. At the end of the follow-up, the median survival time and the survival rate of the patients in the high-expression group were significantly lower than those of the low-expression group $(\mathrm{P}<0.05)$. The high expression of p73 in the peripheral blood of children with WT was positively correlated with the clinical stage of the tumor, and was closely related with the low survival rate of patients.
\end{abstract}

Correspondence to: Dr Cong Xu, Department of Pediatrics (II), People's Hospital of Rizhao, 126 Tai'an Road, Rizhao, Shandong 276800, P.R. China

E-mail: ug45ke@163.com

Key words: Wilms' tumor in children, p73, clinical stage, survival rate

\section{Introduction}

Renal carcinoma in children is rare compared with that in adults. It has been reported that the incidence of renal carcinoma in people under the age of 20 years is $<0.5 \%$, and a great difference exists between the renal carcinoma in children and the renal carcinoma in adults in terms of epidemiology, clinical manifestations, pathological type and prognosis (1). Wilms' tumor (WT), a malignant tumor of the kidneys, is common in children with an incidence of about seven in a million, second only to neuroblastoma in the primary malignant solid tumor of the abdomen in children (2). According to previous data, as a malignant solid mixed tumor is derived from undifferentiated post-renal embryos, WT has a very rapid growth. Blastema cells, immature epithelial tissues, and mesenchymal cells are the components of tumor cells with typical lesions (3), the pathogenesis of which is not clear yet. Therefore, searching for specific target genes and studying the significance of their expression in WT is of great importance.

Highly homologous to the partial sequence of p53 gene, the p73 gene is located at 1p36.2 36.3 and has been proven to be closely related to the occurrence and development of various tumors with overexpression (4). Lowly expressed in normal tissues and excessively expressed in tumor tissues, the p73 is closely related to the proliferation and migration of tumor cells and the prognosis of patients (5). Moreover, it has unique functions in the metabolic regulation (6), neurodevelopment and differentiation (7), as well as the regulation of spermatogenesis and male fertility (8). Many studies have discovered the overexpression of p73 in tumors, such as bladder, ovarian, gastric, lung, thymic epithelial, breast, esophageal and liver cancers (9-11). The study by Mancini et al (12) has found that the expression level of p73, the tumor suppressor gene, has a certain influence on the grade and prognosis of patients with cystic renal cell carcinoma.

Considering that there are only a few studies on the expression of $\mathrm{p} 73$ in the WT in children, in the present study the expression level of p73 mRNA in blood was analyzed, as detected by reverse transcription-quantitative PCR (RT-qPCR), to provide a theoretical basis for the clinical targeted therapy. 
Table I. Primer sequences.

\begin{tabular}{lll}
\hline Genes & \multicolumn{1}{c}{ Forward primers } & \multicolumn{1}{c}{ Reverse primers } \\
\hline p73 & 5'-AGCCACTTGTCACTCAGAACAG-3' & 5'-TCTTACACGAAAAACACGGATG-3' \\
\hline -actin & 5'-GATGAGATTGGCATGGCTTT-3' & 5'-CACCTTCACCGTTCCAGTTT-3' \\
\hline
\end{tabular}

\section{Subjects and methods}

General information. Fifty patients (29 males and 21 females, aged from 13 to 65 months) diagnosed with WT in the People's Hospital of Rizhao (Rizhao, China), from July 2013 to January 2015, were enrolled as the study group, including 15 cases in stage I, 17 cases in stage II, 11 cases in stage III, and 7 cases in stage IV, according to the clinical stage standardized by the NWTS-5 (US WT Research Collaboration Group). In the study group there were 12 cases of unfavorable histopathology (UH) and 38 cases of favorable histopathology $(\mathrm{FH})$, according to the pathological classification. Also, there were 10 patients with lymphatic metastasis, 13 patients with vascular invasion, 5 patients with failed treatment and 45 patients with successful treatment, according to the treatment outcome. Twenty healthy children (13 males and 7 females, aged from 15 to 68 months) with similar age and sex, who received health examinations in the same hospital during the same period, were enrolled as the control group.

The inclusion criteria were: children confirmed by pathology as WT patients; patients with complete clinical and pathological data; patients with no chemotherapy or radiotherapy; patients who received examination of liver and kidney function, blood routine examination and other necessary examinations before operation; patients with normal body mass index.

The exclusion criteria were: patients with other malignant tumors; patients with severe congenital heart disease; patients with liver dysfunction and severe organ diseases; patients with autoimmune diseases; patients who did not cooperate with the medical examinations; patients whose family members refused to sign the informed consent.

The study was approved by the Ethics Committee of the People's Hospital of Rizhao and the research subjects' family members signed a complete informed consent form after receiving details on the experimental contents.

Serum collection. After diagnosis, $2 \mathrm{ml}$ of peripheral blood were collected from WT children, with an empty stomach in the morning, and placed in an anticoagulation tube to be sent to the laboratory; and $2 \mathrm{ml}$ of fasting venous blood were taken from the children of the control group in the morning, on the day of the physical examination. After coagulation for $60 \mathrm{~min}$ at $20-25^{\circ} \mathrm{C}$, the blood samples of the two groups were centrifuged at $2,600 \mathrm{x} \mathrm{g}$ for $10 \mathrm{~min}$ at $4^{\circ} \mathrm{C}$ to obtain the supernatant liquid that was kept at $-80^{\circ} \mathrm{C}$ to be tested (repeated freezing and thawing was avoided as far as possible).

Experimental reagents and instruments. RNAiso Plus kit (Takara Biotechnology Co., Ltd., Dalian, China); Two-Step
Reverse Transcription kit (Takara Bio, Inc., Otsu, Japan); SYBR-Green qPCR Master Mix kit (Thermo Fisher Scientific, Inc., Waltham, MA, USA); UV spectrophotometry instrument (Bio-Rad Laboratories, Inc., Hercules, CA, USA); RT-qPCR kit and StepOnePlus real-time PCR instrument (both from Thermor Fisher Scientific, Inc.).

Primers. The primers of this study were designed by the Primer Premier 5.0 design software (Premier Biosoft, Inc., Palo Alto, CA, USA) and were generated by Tianjin Saier Biotechnology Co., Ltd. (Tianjin, China). The sequences of the reference gene $\beta$-actin (13) and the p73 are shown in Table I.

Experimental procedures. The specimens were dissolved at $4^{\circ} \mathrm{C}$ overnight, and were transferred to a new centrifuge tube after mixing by oscillation. Total RNA was extracted using the RNAiso Plus kit in strict accordance with the manufacturer's instructions; the purity and concentration of the RNA were measured by UV spectrophotometry; the RNA integrity was detected by the agarose gel electrophoresis. Then the method of Two-Step reverse transcription was performed to transfer RNA to cDNA: i) first step: removal of the genomic DNA. The DNase I digestion of the template before the reverse transcription was performed to eliminate the pollution of the genome. The reaction system was $10 \mu \mathrm{l}$ in total, with $1 \mu \mathrm{g}$ of RNA, and the reaction conditions were: $42^{\circ} \mathrm{C}$ for $2 \mathrm{~min}$. Then, the system was quickly put on ice for cooling and kept at $4^{\circ} \mathrm{C}$. ii) Second step: reverse transcription reaction. The above extracted total RNA was reversely transcribed into cDNA, and then frozen at $-20^{\circ} \mathrm{C}$. The reaction system was $20 \mu 1$ in total, $37^{\circ} \mathrm{C}$ for $15 \mathrm{~min}$, afterwards $85^{\circ} \mathrm{C}$ for $5 \mathrm{sec}$, and then the system was kept at $4^{\circ} \mathrm{C}$. qPCR was subsequently performed using the SYBR-Green qPCR Master Mix. RT-qPCR: the sample was kept on ice away from light, and the reaction system was $20 \mu 1$ in total, with $3 \mu \mathrm{l}$ of the template, $0.8 \mu \mathrm{l}(10 \mu \mathrm{M})$ of the forward primer and $0.8 \mu \mathrm{l}(10 \mu \mathrm{M})$ of the reverse primer of $\mathrm{p} 73$, and $0.8 \mu \mathrm{l}$ $(10 \mu \mathrm{M})$ of the forward primer and $0.8 \mu 1(10 \mu \mathrm{M})$ of the reverse primer of $\beta$-actin. The reaction conditions were 40 cycles of $95^{\circ} \mathrm{C}$ for $30 \mathrm{sec}, 95^{\circ} \mathrm{C}$ for $5 \mathrm{sec}, 60^{\circ} \mathrm{C}$ for $34 \mathrm{sec}$, and the dissolution curve: $95^{\circ} \mathrm{C}$ for $15 \mathrm{sec}, 60^{\circ} \mathrm{C}$ for $1 \mathrm{~min}$, $95^{\circ} \mathrm{C}$ for $15 \mathrm{sec}, 60^{\circ} \mathrm{C}$ for $15 \mathrm{sec}$. The product of p73 was $124 \mathrm{bp}$ in length and the product of $\beta$-actin was $100 \mathrm{bp}$ in length. The configuration and operation of the above reaction systems were strictly in accordance with the respective protocols. Three independent experiments were performed to record the $\mathrm{Cq}$ value and the number of cycles required for the fluorescence signal to reach the set threshold from the background, and the data were analyzed using the formula: Relative quantity $(\mathrm{RQ})=2^{-\mathrm{ACq}}(14)$. The control group in this experiment served as the internal control. 
Table II. Comparison of the baseline data between the study and the control group $[\mathrm{n}(\%)] /(\mathrm{mean} \pm \mathrm{SD})$.

\begin{tabular}{|c|c|c|c|c|}
\hline Clinical data & Study group $(n=50)$ & Control group $(n=20)$ & $\chi^{2} / \mathrm{t}$ & P-value \\
\hline Sex & & & 0.292 & 0.589 \\
\hline Male & $29(58.0)$ & $13(65.0)$ & & \\
\hline Female & $21(42.0)$ & $7(35.0)$ & & \\
\hline Age (years) & & & 1.072 & 0.301 \\
\hline$\leq 3$ & $31(62.0)$ & $15(75.0)$ & & \\
\hline$>3$ & $19(38.0)$ & $5(25.0)$ & & \\
\hline Body mass index $\left(\mathrm{kg} / \mathrm{m}^{2}\right)$ & $14.5 \pm 7.09$ & $14.1 \pm 6.86$ & 0.689 & 0.058 \\
\hline $\mathrm{Hb}(\mathrm{gm} / \mathrm{dl})$ & $11.48 \pm 1.69$ & $11.67 \pm 1.87$ & -1.102 & 0.499 \\
\hline PLT $\left(x 10^{9} / 1\right)$ & $151.89 \pm 20.98$ & $156.18 \pm 21.65$ & -1.564 & 0.577 \\
\hline WBC $\left(\times 10^{9} / 1\right)$ & $7.38 \pm 2.51$ & $7.15 \pm 2.32$ & 0.602 & 0.304 \\
\hline $\operatorname{RBC}\left(\times 10^{12} / 1\right)$ & $4.32 \pm 0.49$ & $4.28 \pm 0.43$ & 0.185 & 0.356 \\
\hline ALT (U/l) & $21.97 \pm 10.04$ & $21.36 \pm 9.86$ & 1.996 & 0.226 \\
\hline AST (U/l) & $18.87 \pm 7.01$ & $18.12 \pm 6.46$ & 2.542 & 0.145 \\
\hline
\end{tabular}

$\mathrm{Hb}$, hemoglobin; PLT, platelet; WBC, white blood cell; RBC, red blood cell; ALT, alanine transaminase; AST, aspartate transaminase.

Follow-up and observation indicators. After the end of treatment, follow-up for patients with WT was carried out by patients' revisiting the hospital and telephone surveys, during which prompt treatment and strengthened examinations were provided for those patients with reoccurrence of the disease. The final follow-up time and situation were recorded for patients lost to follow-up and the date of death was recorded in detail for patients who died after the treatment, for the completion of the prognostic data. Follow-up deadline was February 1, 2018. Survival time was recorded from the first day of diagnosis after admission to the day of death or follow-up deadline. The expression of p73 protein in each group, its relationship with the clinicopathological features and the clinical stages, as well as the survival rates were observed.

Statistical analysis. Statistical analysis of the experimental data was carried out using SPSS 17.0 statistical software (Tianjin KSoft Tech. Co., Ltd., Tianjin, China). Enumeration data were expressed as $\mathrm{n}(\%)$ and their comparison between groups was made using Chi-square $\left(\chi^{2}\right)$ test. Measurement data were expressed as mean \pm SD and their comparison between groups was made using t-test. Comparisons of the mean of multiple groups were made using one-way analysis of variance (ANOVA) with Least Significant Difference post hoc test. The correlation between the clinical staging and p73 expression in the peripheral blood was analyzed by Spearman's correlation coefficient. Survival curves were generated based on the Kaplan-Meier analysis and log-rank test was used for their comparison. $\mathrm{P}<0.05$ was considered to indicate a statistically significant difference.

\section{Results}

Comparison of clinical general data. No statistical difference was detected in the comparison of the general clinical baseline data between the two groups including sex, age, body
Table III. Comparison of p73 expression levels in the blood of the study and the control group (mean $\pm \mathrm{SD}$ ).

\begin{tabular}{lcc}
\hline Group & No. of cases & $\mathrm{p} 73$ \\
\hline Study group & 50 & $4.04 \pm 0.79$ \\
Control group & 20 & $1.57 \pm 1.12$ \\
$\mathrm{t}$ & & 11.44 \\
P-value & & $<0.01$ \\
\hline
\end{tabular}

mass index, hemoglobin (Hb), platelet (PLT) count, white blood cell (WBC) count, red blood cell (RBC) count, alanine transaminase (ALT) and aspartate transaminase (AST) (P>0.05) (Table II).

Comparison of the expression levels of $p 73$ in the peripheral blood of the two groups. As shown in Table III, the expression level of p73 in the blood of the study group (4.04 \pm 0.79$)$ was significantly higher than that of the control group $(1.57 \pm 1.12)$ and the difference was statistically significant $(\mathrm{t}=11.44, \mathrm{P}<0.01)$.

Relationship between the expression levels of p73 in the peripheral blood and the clinicopathological features of WT patients. According to Table IV, the differences of the expression levels of $\mathrm{p} 73$ in the peripheral blood among WT patients of different age, sex, or vascular invasion situation were not statistically significant $(\mathrm{P}>0.05)$; while patients with different tumor size, pathological type, lymph node metastasis situation, or treatment outcome had significantly different p73 expression levels in the peripheral blood $(\mathrm{P}<0.05)$.

Comparison of p73 expression levels in the peripheral blood of patients with WT in different clinical stages. The clinical 
Table IV. Relationship between the expression levels of p73 in the peripheral blood and the clinicopathological features of WT patients (mean $\pm \mathrm{SD}$ ).

\begin{tabular}{lcccc}
\hline $\begin{array}{l}\text { Clinicopathological } \\
\text { features }\end{array}$ & $\begin{array}{c}\text { No. of } \\
\text { cases }\end{array}$ & $\mathrm{p} 73$ & $\mathrm{t}$ & P-value \\
\hline Sex & & & 1.611 & 0.112 \\
$\quad$ Male & 29 & $3.34 \pm 0.95$ & & \\
$\quad \begin{array}{l}\text { Female } \\
\text { Age (years) }\end{array}$ & 21 & $3.21 \pm 1.07$ & & \\
$\leq 3$ & & & & \\
$>3$ & 31 & $3.37 \pm 0.89$ & -0.110 & 0.052 \\
Tumor size (cm) & 19 & $3.29 \pm 1.21$ & & \\
$\leq 10$ & & & -3.439 & $0.009^{\mathrm{a}}$ \\
$>10$ & 35 & $2.98 \pm 0.87$ & & \\
Pathological type & 15 & $3.96 \pm 0.52$ & & \\
FH & & & -2.213 & $0.008^{\mathrm{a}}$ \\
UH & 38 & $2.95 \pm 0.87$ & & \\
Lymph node metastasis & 12 & $3.86 \pm 0.52$ & & \\
$\quad$ Yes & & & 7.808 & $0.004^{\mathrm{a}}$ \\
$\quad$ No & 10 & $4.87 \pm 0.23$ & & \\
Vascular invasion & 40 & $3.14 \pm 0.87$ & & \\
$\quad$ Yes & & & 0.624 & 0.052 \\
No & 13 & $3.35 \pm 0.37$ & & \\
Treatment outcome & 37 & $3.46 \pm 0.67$ & & \\
Failure & & & -1.182 & $0.032^{\mathrm{a}}$ \\
Success & 5 & $4.63 \pm 0.42$ & & \\
\hline & 45 & $3.01 \pm 1.01$ & & \\
\hline
\end{tabular}

${ }^{a} \mathrm{P}<0.05$, statistically significant difference. WT, Wilms' tumor; $\mathrm{FH}$, favorable histopathology; UH, unfavorable histopathology.

Table V. Comparison of $\mathrm{p} 73$ expression in the peripheral blood of patients with WT in different clinical stages (mean \pm SD).

\begin{tabular}{lcc}
\hline Clinical stage & No. of cases & $\mathrm{p} 73$ \\
\hline I & 15 & $2.05 \pm 0.98$ \\
II & 17 & $2.99 \pm 0.65^{\mathrm{a}}$ \\
III & 11 & $3.76 \pm 0.43^{\mathrm{a}, \mathrm{b}}$ \\
IV & 7 & $4.48 \pm 0.33^{\mathrm{a}-\mathrm{c}}$ \\
F & & 23.54 \\
P-value & & $<0.01$ \\
\hline
\end{tabular}

${ }^{\mathrm{a}} \mathrm{P}<0.05$, statistically significant difference when compared with stage I. ${ }^{b} \mathrm{P}<0.05$, statistically significant difference when compared with stage II. ${ }^{\mathrm{C}} \mathrm{P}<0.05$, statistically significant difference when compared with stage III. WT, Wilms' tumor.

stages ranged from I to IV. According to the specific expression level of p73 in blood (Table V), the expression level of p73 in the peripheral blood of WT patients was in a progressive increase with the change of clinical stage. The expression levels of p73 among different clinical stages were significantly

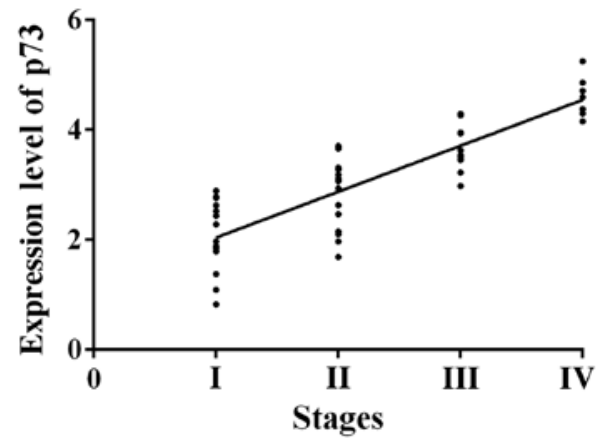

Figure 1. Correlation between the clinical stage and the expression of p73 mRNA in children with WT. Spearman's correlation analysis showed that the expression level of p73 mRNA in the peripheral blood of WT patients progressively increased with changes of the clinical stage, with a positive correlation between them $(\mathrm{r}=0.7230, \mathrm{P}<0.01)$. WT, Wilms' tumor.

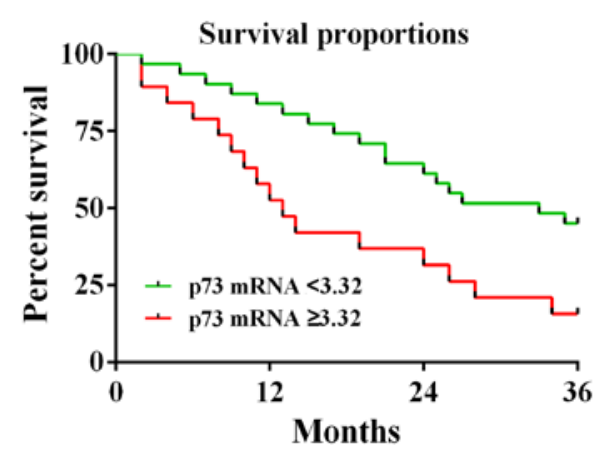

Figure 2. Relationship between p73 mRNA expression in the peripheral blood of WT patients and the survival rate of patients. The median survival time of the low-expression group (30 months) was found to be much longer compared with that of the high-expression group (13.5 months) $(\mathrm{P}<0.05)$. The 3 -year follow-up revealed the relationship between the high expression of p73 and the low survival rate of patients since the survival rate of the patients in the high-expression group $(15.8 \%)$ was significantly lower than that of the low-expression group (45.2\%). WT, Wilms' tumor.

different $(\mathrm{F}=23.54, \mathrm{P}<0.01)$. The Spearman's correlation analysis showed that the correlation between the expression level of $\mathrm{p} 73$ and the clinical stages is positive $(\mathrm{r}=0.7230$, $\mathrm{P}<0.01$ ) (Fig. 1).

Relationship between the 773 expression level in the peripheral blood and the survival rate of children with WT. According to the survival data of children in the study group and taking the mean value of the expression of p73 (3.32) as the boundary, the patients with $\mathrm{p} 73$ expression value $<3.32$ were considered as the low-expression group (31 children; median survival time, 30 months) and the patients with $p 73$ expression value $\geq 3.32$ were considered as the high-expression group (19 children; median survival time, 13.5 months) (Fig. 2). The 3-year follow-up revealed the relationship between the high expression of p73 and the low survival rate of patients. The survival rate of the patients in the high-expression group (15.8\%) was significantly lower than that of the low-expression group (45.2\%).

\section{Discussion}

WT, a malignant tumor common in children 2-3 years of age, has common clinical symptoms, such as abdominal masses, 
irritability, weight loss and anhelation, and a certain mortality rate $(15,16)$. The development in medical technology has offered surgical treatment as the main method for treating WT in children. It has good efficacy, but causes large trauma and many adverse reactions harmful to the recovery of children (17). Therefore, the study of the molecular mechanism of WT is particularly important. Since the molecular research on the development mechanism of WT is still unclear, the 'nephrogenic rest' theory (18) has been confirmed to a certain degree, which suggests that the embryonic renal tissue in some individual renal tissues may be the pre-neoplastic lesions of the WT. Worldwide studies on many genes have been found to be related to the occurrence and development of WT, such as WT1, WT2, WTX, WT3, WT4 (FWT1), p53, p16, tumor synechia and other molecular genes $(19,20)$, but studies on their mechanism of action are rare. This study found that p73 plays an important role in the WT in children.

As part of the p53 family, with different kinds of promoter transcription and alternative splicing, p73 can produce $>10$ different subtypes, collectively called DNp73 or $\Delta$ TAp73 (21), that play an important role in the expression of human tumors. The DNp73 is different from p53 in function due to the significant difference in their structures, while the $\Delta$ TAp73 has similar functions as p53 in inhibiting tumor and promoting apoptosis (22). p73 gene is characterized by the dual feature of tumor suppressor and carcinogenesis because of the co-expression and mutual intervention of different subtypes of p73 and p53 (21), proved by related studies to be possibly involved in the proliferation, metastasis, and invasion of tumor cells. The mechanism of action of p73 may be as follows: i) as one of the direct transcriptional target genes of E2F-1 which mainly regulates cell proliferation and is overexpressed in various cell lines, p73 can have induced expression by E2F-1 (23). The study by Ozono et al (24) has shown that the ERE73s located in the TAP73 promoter can detect the abnormal expression of E2F in the normal human fibroblasts, likely in joint action with the TAP73 to induce the increase of DNp73 mRNA expression in the body. ii) DNp73, a subtype of p73, is involved in the proliferation, metastasis, and invasion of tumor cells, mainly by stimulating the expression of Cola1 and PAI-1 receptors in the TGF- $\beta$ signaling pathway (25). Certain relations between the occurrence, development, and prognosis of various malignant tumors and the p73 are recognized $(26,27)$.

In the present study, the study and control groups were not obviously different in the clinical general data, according to the comparison between the two groups $(\mathrm{P}>0.05)$, protecting this experiment from the interference by some basic clinical indicators. The analysis of the relationship between the expression level of p73 in the peripheral blood and the clinicopathological features of WT revealed the differences of the p73 expression levels among patients with different tumor sizes, pathological types, lymph node metastasis, and treatment outcomes $(\mathrm{P}<0.05)$, suggesting the important role of p73 in the occurrence and development of WT (28). This study found that the expression level of p73 in the peripheral blood of the study group $(4.04 \pm 0.79)$ was much higher than that of the control group $(1.57 \pm 1.12)$, indicating the high concentration of $\mathrm{p} 73$ in the patient's serum. Related studies (29) have pointed out that the p73 gene is highly expressed in tumors in the main form of DNp73. In addition, some studies (30) have confirmed the frequent increase in the expression of the subtype, DNp73 in cervical cancer, but the present study aimed at the WT. Due to the significantly higher expression of p73 in the peripheral blood of patients in the study group, compared with that in the control group, the assumption was drawn that the expression of p73 in the peripheral blood of patients with WT was mainly in the form the DNp73, and the overexpression of p73 in tumor patients was also indirectly indicated (4). The present study revealed the significantly positive correlation between the expression level of p73 and the clinical stage by comparing the expression of p73 in the peripheral blood of patients in different clinical stages $(\mathrm{r}=0.7230, \mathrm{P}<0.01)$, suggesting that $\mathrm{p} 73$ is of great significance for the clinical stage of WT in children. The expression level of p73 in patients progressively increased with the increase of tumor malignancy, indicating that $\mathrm{p} 73$ may be involved in the proliferation, invasion and metastasis of tumor cells (22). Analysis of the relationship between the expression of p73 in the peripheral blood of patients with WT and the survival rate of patients showed that the survival rate of the high-expression group is significantly lower than that of the low-expression group $(\mathrm{P}<0.05)$, presenting the relationship between the low survival rate of patients and the high expression of p73. Also, in literature it has been stated that patients with highly expressed p73 have low survival rate (25). However, the absolute relationship between the high expression of p73 in patients with WT and the poor survival rate in patients has not been verified by literature.

The results of the present study have certain reliability in consideration of its comprehensive analysis of the clinical baseline data of the two groups, the relationship between the expression level of p73 in the peripheral blood and the clinicopathological features of WT, the comparison of the expression levels of p73 in the peripheral blood between the two groups, the comparison of the expression of p73 in the peripheral blood among patients with different clinical stages, and the relationship between the expression of p73 in the peripheral blood of WT patients, and the survival rate of patients. However, this study was retrospective, which brought certain limitations. Thus, more research subjects are needed in a future study to better serve clinical treatment.

In summary, the finding that p73 is highly expressed in WT in children provides guiding significance for the clinical diagnosis and prognosis of WT in children.

\section{Acknowledgements}

Not applicable.

\section{Funding}

No funding was received.

\section{Availability of data and materials}

The datasets used and/or analyzed during the current study are available from the corresponding author on reasonable request.

\section{Authors' contributions}

YD drafted the manuscript. YD and XG were responsible for the serum collection and PCR. XL and JL collected and 
analyzed the general data of patients. NL and CX contributed to the follow-up and the analysis of the observation indicators. The final version was read and approved by all authors.

\section{Ethics approval and consent to participate}

The present study was approved by the Ethics Committee of the People's Hospital of Rizhao (Rizhao, China). Patients who participated in this study, had complete clinical data. Signed informed consents were obtained from the parents of the child patients/or the guardians.

\section{Patient consent for publication}

Not applicable.

\section{Competing interests}

The authors declare that they have no competing interests.

\section{References}

1. Saltzman AF, Carrasco A Jr, Weinman J, Meyers ML and Cost NG: Initial imaging for pediatric renal tumors: An opportunity for improvement. J Urol 199: 1330-1336, 2018.

2. Bozlu G and Çitak EÇ: Evaluation of renal tumors in children. Turk J Urol 44: 268-273, 2018.

3. Tian F, Yourek G, Shi X and Yang Y: The development of Wilms tumor: From WT1 and microRNA to animal models. Biochim Biophys Acta 1846: 180-187, 2014.

4. Zhang Y, Young A, Zhang J and Chen X: P73 tumor suppressor and its targets, p21 and PUMA, are required for Madin-Darby canine kidney cell morphogenesis by maintaining an appropriate level of epithelial to mesenchymal transition. Oncotarget 6 : 13994-14004, 2015.

5. Kurian JJ, Jehangir S and Korula A: Multiloculated cystic renal tumors of childhood: Has the final word been spoken? J Indian Assoc Pediatr Surg 23: 22-26, 2018.

6. Cutruzzolà F, Avigliano L and Candi E: p73 keeps metabolic control in balance. Cell Cycle 13: 179-180, 2014.

7. Killick R, Niklison-Chirou M, Tomasini R, Bano D, Rufini A, Grespi F, Velletri T, Tucci P, Sayan BS, Conforti F, et al: p73: A multifunctional protein in neurobiology. Mol Neurobiol 43: 139-146, 2011.

8. Inoue S, Tomasini R, Rufini A, Elia AJ, Agostini M, Amelio I, Cescon D, Dinsdale D, Zhou L, Harris IS, et al: TAp73 is required for spermatogenesis and the maintenance of male fertility. Proc Natl Acad Sci USA 111: 1843-1848, 2014.

9. Ye B, Wang X, Yang Z, Sun Z, Zhang R, Hu Y, Lu Y and Du J: p53 and p73 expression in esophageal carcinoma correlate with clinicopathology of tumors. Hepatogastroenterology 59: 2192-2195, 2012

10. Arisawa A, Watanabe Y, Tanaka H, Takahashi H, Matsuo C, Fujiwara T, Fujiwara M, Fujimoto Y and Tomiyama N: Comparative study of pulsed-continuous arterial spin labeling and dynamic susceptibility contrast imaging by histogram analysis in evaluation of glial tumors. Neuroradiology 60: 599-608, 2018.

11. Moll UM and Slade N: p63 and p73: roles in development and tumor formation. Mol Cancer Res 2: 371-386, 2004.

12. Mancini P, Angeloni A, Risi E, Orsi E and Mezi S: Standard of care and promising new agents for triple-negative metastatic breast cancer. Cancers (Basel) 6: 2187-2223, 2014.
13. Murphy AJ, Pierce J, de Caestecker C, Taylor C, Anderson JR, Perantoni AO, de Caestecker MP and Lovvorn HN III: SIX2 and CITED1, markers of nephronic progenitor self-renewal, remain active in primitive elements of Wilms' tumor. J Pediatr Surg 47: 1239-1249, 2012.

14. Livak KJ and Schmittgen TD: Analysis of relative gene expression data using real-time quantitative PCR and the 2(-Delta Delta C(T)) method. Methods 25: 402-408, 2001.

15. Ishibashi K, Haber T, Breuksch I, Gebhard S, Sugino T, Kubo H, Hata J, Koguchi T, Yabe M, Kataoka M, et al: Overriding TKI resistance of renal cell carcinoma by combination therapy with IL-6 receptor blockade. Oncotarget 8: 55230-55245, 2017.

16. Cutruzzula P, Cahn D, Kivlin D, Tong C, Edwards D and Amster M: A review of translocation $\mathrm{t}(6 ; 11)$ renal cell carcinoma tumors in the adult patient. Curr Urol 10: 69-71, 2017.

17. Schultz KAP, Rednam SP, Kamihara J, Doros L, Achatz MI, Wasserman JD, Diller LR, Brugières L, Druker $\mathrm{H}$, Schneider KA, et al: Pten, dicer1, fh, and their associated tumor susceptibility syndromes: Clinical features, genetics, and surveillance recommendations in childhood. Clin Cancer Res 23: e76-e82, 2017.

18. Al-Hussain T, Ali A and Akhtar M: Wilms tumor: An update. Adv Anat Pathol 21: 166-173, 2014.

19. Zitzmann F, Mayr D, Berger M, Stehr M, von Schweinitz D, Kappler R and Hubertus J: Frequent hypermethylation of a CTCF binding site influences Wilms tumor 1 expression in Wilms tumors. Oncol Rep 31: 1871-1876, 2014.

20. Franken J, Lerut E, Van Poppel H and Bogaert G: p53 immunohistochemistry expression in Wilms tumor: A prognostic tool in the detection of tumor aggressiveness. J Urol 189: 664-670, 2013

21. Melino G, De Laurenzi V and Vousden KH: p73: Friend or foe in tumorigenesis. Nat Rev Cancer 2: 605-615, 2002.

22. Song DJ, Yue LF, Zhang D, Yang HY, Fan YX, Yue M, Pei H and Wang JX: Relationship between mRNA expression and promoter methylation status of p73 gene in peripheral blood among children with Wilms' tumor. Zhongguo Dang Dai Er Ke Za Zhi 15: 638-643, 2013 (In Chinese).

23. McKeon F and Melino G: Fog of war: The emerging p53 family. Cell Cycle 6: 229-232, 2007.

24. Ozono E, Komori H, Iwanaga R, Tanaka T, Sakae T, Kitamura H, Yamaoka S and Ohtani K: Tumor suppressor TAp73 gene specifically responds to deregulated E2F activity in human normal fibroblasts. Genes Cells 17: 660-672, 2012.

25. Fuchs J: Surgical concepts in the treatment of Wilms tumor: An update. Urologe A 54: 1784-1791, 2015 (In German).

26. Al-Daghmin A, Alhamss S, Al-Qasem K, Al-Najjar H, Al-Smadi K, Olaimat A and Al-Halbouni L: Patterns of management of translocation renal cell carcinoma. Turk J Urol 44: 467-472, 2018.

27. Pan X, Quan J, Zhao L, Li W, Wei B, Yang S and Lai Y: Xp11.2 translocation renal cell carcinoma with TFE3 gene fusion: A case report. Mol Clin Oncol 8: 83-85, 2018.

28. Yong M, Yang L, Suyila Q, Han W, Yuan H, Zhao C and Su X: Expression and clinical implications of $\mathrm{P} 53, \mathrm{P} 63$, and $\mathrm{P} 73$ protein in malignant tumor of the parotid gland. Turk J Med Sci 44: 875-882, 2014

29. Ishimoto O, Kawahara C, Enjo K, Obinata M, Nukiwa T and Ikawa S: Possible oncogenic potential of DeltaNp73: A newly identified isoform of human p73. Cancer Res 62: 636-641, 2002.

30. Inoue K and Fry EA: Alterations of p63 and p73 in human cancers. Subcell Biochem 85: 17-40, 2014.

This work is licensed under a Creative Commons Attribution-NonCommercial-NoDerivatives 4.0 International (CC BY-NC-ND 4.0) License. 